\title{
Optimal on/off scheme for all-optical switching
}

\author{
Kristensen, Philip Trøst; Heuck, Mikkel; Mørk, Jesper
}

Published in:

Advanced Photonics Congress

Publication date:

2012

Document Version

Publisher's PDF, also known as Version of record

Link back to DTU Orbit

Citation (APA):

Kristensen, P. T., Heuck, M., \& Mørk, J. (2012). Optimal on/off scheme for all-optical switching. In Advanced Photonics Congress (pp. IM3B.3). Optical Society of America.

\section{General rights}

Copyright and moral rights for the publications made accessible in the public portal are retained by the authors and/or other copyright owners and it is a condition of accessing publications that users recognise and abide by the legal requirements associated with these rights.

- Users may download and print one copy of any publication from the public portal for the purpose of private study or research.

- You may not further distribute the material or use it for any profit-making activity or commercial gain

- You may freely distribute the URL identifying the publication in the public portal

If you believe that this document breaches copyright please contact us providing details, and we will remove access to the work immediately and investigate your claim. 


\title{
Optimal on/off scheme for all-optical switching
}

\author{
Philip Trøst Kristensen, Mikkel Heuck and Jesper Mørk \\ DTU Fotonik, Technical University of Denmark, Ørsteds plads 345, DK-2800 Kgs. Lyngby, Denmark \\ ptkr@fotonik.dtu.dk
}

\begin{abstract}
We present a two-pulsed on/off scheme based on coherent control for fast switching of the optical energy in a micro cavity and use calculus of variations to optimize the switching in terms of energy.
\end{abstract}

(C) 2012 Optical Society of America

OCIS codes: $000.3860,250.6715$.

\section{Introduction}

The high energy density in optical micro cavities may lead to pronounced non-linear effects from material responses such as the Kerr effect. This provides a mechanism for all-optical switching in which a control pulse shifts the resonance of an optical cavity to allow the transmission of a signal pulse at a different frequency, as illustrated in Fig.1. Optical fields carry a phase that can be utilized for additional engineering of the coupling in and out of cavities. It has recently been illustrated how this type of coherent control can improve the energy efficiency [1] and opens possibilities for switching controlled entirely by the phase of the input light [2]. Regardless of the particular choice of operating principle, there is a need for fast switching of the energy in the cavity between the values $U_{\text {on }}$ and $U_{\text {off }}$, corresponding to the "on" and "off" state of the switch, since residual energy may lead to unwanted patterning effects [3]. We show, that within the limits of our model, coherent control provides an approach for arbitrarily fast switching of the energy in the cavity. Therefore, from an optimization point of view, the total energy required for a given switching action is a more interesting figure of merit than the switching speed. For an example material system with a Kerr non-linearity we solve this problem analytically using calculus of variations and show that the optimal scheme is slightly different from our initial (constant power) approach. We illustrate the impact of our findings with direct numerical evaluation of the wave equation and find excellent agreement between the analytical and the numerical results.
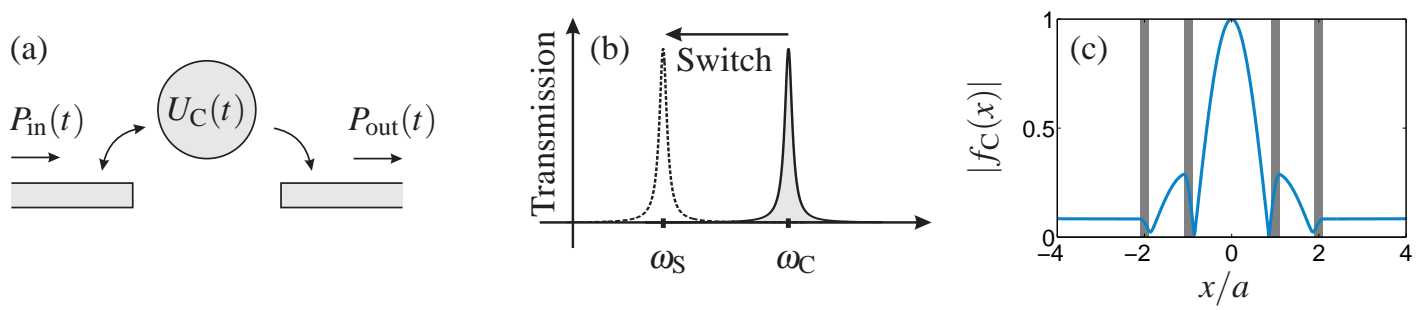

Fig. 1: (a): Diagram of typical waveguide-cavity-waveguide system with input/output powers $P_{\text {in/out }}(t)$ indicated along with the cavity energy $U_{\mathrm{C}}(t)$. (b): Typical transmission profile for the system in (a) with resonance frequency $\omega_{\mathrm{C}}$. Non-linear effects may change the resonance frequency to transmit a signal at the frequency $\omega_{\mathrm{S}}$. (c): Example cavity system in a finite one-dimensional photonic crystal with lattice constant $a$ made from barriers of permittivity $\varepsilon_{\mathrm{r}}=13$ and radius $r=0.1 a$ in air. Blue solid curve shows the fundamental cavity mode.

\section{Coupled mode theory and constant power approach}

To analyze coherently controlled switching, we use coupled mode theory [4] (CMT) to set up equations for the energy and the phase of the field in the cavity as

$$
\begin{aligned}
\frac{\mathrm{d}}{\mathrm{d} t} U_{\mathrm{C}}(t) & =-\frac{2}{\tau_{\mathrm{C}}} U_{\mathrm{C}}(t)+2 \sqrt{U_{\mathrm{C}}(t)} \sqrt{\frac{P_{\text {in }}(t)}{\tau_{\mathrm{C}}}} \cos \left(\varphi(t)-\varphi_{\mathrm{S}}(t)\right), \\
\frac{\mathrm{d}}{\mathrm{d} t} \varphi(t) & =-\Delta \omega+F_{\mathrm{NL}} U_{\mathrm{C}}(t)-\sqrt{\frac{1}{U_{\mathrm{C}}(t)}} \sqrt{\frac{P_{\mathrm{in}}(t)}{\tau_{\mathrm{C}}}} \sin \left(\varphi(t)-\varphi_{\mathrm{S}}(t)\right),
\end{aligned}
$$


in which $1 / \tau_{\mathrm{C}}$ represents the total decay rate of the field, and $\Delta \omega=\left(\omega_{\mathrm{C}}-\omega_{\mathrm{S}}\right)$, where $\omega_{\mathrm{S}}$ and $\varphi_{\mathrm{S}}$ is the frequency and phase of the input light, respectively. $F_{\mathrm{NL}}>0$ is the effective non-linear Kerr coefficient that accounts for the material non-linearity as well as the field distribution in the cavity. This form of the CMT equations follows directly from the standard formulation in Ref. [4] and is convenient when analyzing effects of coherent control on the switching action. From Eq. (1), the largest possible instantaneous rate of change of the cavity energy is found when $\varphi-\varphi_{\mathrm{S}}=p \pi$ with integer $p$. This suggests a two-pulsed on/off switching scheme in which the input power is (piecewise) constant and we vary only the phase. For switching on (off), we choose $p$ even (odd) and set $P_{\text {in }}=P_{\text {on }}\left(P_{\text {in }}=P_{\text {off }}\right)$. For $U_{\mathrm{c}}(0)=0$ we find

Switch on:

$$
U_{\mathrm{C}}(t)=P_{\mathrm{on}} \tau_{\mathrm{C}}\left(e^{-t / \tau_{\mathrm{C}}}-1\right)^{2}
$$

The energy in the cavity thus increases, at a rate set by $\tau_{\mathrm{C}}$, towards the maximum energy of $P_{\mathrm{on}} \tau_{\mathrm{c}}$. In a similar way, for switching off, we set $U_{\mathrm{c}}(0)=U_{\text {on }}$ to find

Switch off: $\left.\quad U_{\mathrm{C}}(t)=P_{\text {off }} \tau_{\mathrm{C}}\left(\sqrt{\frac{U_{\text {on }}}{P_{\text {off }} \tau_{\mathrm{C}}}}+1\right) e^{-t / \tau_{\mathrm{C}}}-1\right)^{2}$.

Importantly, the transition times for both the switch on and switch off actions can be made shorter by simply applying a larger input power, as illustrated in Fig. 2 (a) for the switch on. For the switch off, interference effects act to decrease the energy in the cavity faster than the cavity decay rate, see Fig. 3. In Eqs. (3) and (4), the respective phase relations have been assumed to be satisfied. For linear problems at resonance, this is satisfied for constant input phase and $\varphi_{\mathrm{S}}^{\text {off }}=\varphi_{\mathrm{S}}^{\mathrm{on}}+\pi$, cf. Eq. (2). In the general case, integration of Eq. (2) provides the required change in input phase.

This two-pulsed approach to fast switching by coherent control may be immediately applied to a wide range of systems described by Eqs. (1) and (2). As an explicit (linear) example we set $F_{\mathrm{NL}}=0$ and consider the one-dimensional model of a waveguide-cavity-waveguide system in Fig. 1 (c), for which a time-dependent finite element (FEM) based model provides fast and very accurate reference solutions. We choose a model switch on/switch off action where $U_{\text {off }}=0$ and $U_{\text {on }}=P_{0} \tau_{\mathrm{C}}$, and use two input pulses of width $T=\tau_{\mathrm{C}} / 2$ with the second (switch off) following directly after the first (switch on). The required input powers in the two pulses are derived from Eqs. (3) and (4), and for this practical example we use super Gaussian pulse shapes of the form $P_{\text {in }}=P_{\text {on/off }} \exp \left\{-\left(t-t_{0}\right) / \tau_{\mathrm{C}}\right\}^{4 N}$, with $N=10$. Fig. 2 (b) shows the input pulses as well as the CMT solution and FEM calculation of the time dependent cavity energy. There is a good agreement between the two solutions, except for a number of minor oscillations in the FEM result which are caused by frequency components outside the validity of the simple single-resonance model implied in CMT. Overall, the two-pulsed on/off scheme works as intended to perform the desired switching action, clearly illustrating the potential of the CMT model and motivating further analysis of the two-pulse switching scheme.
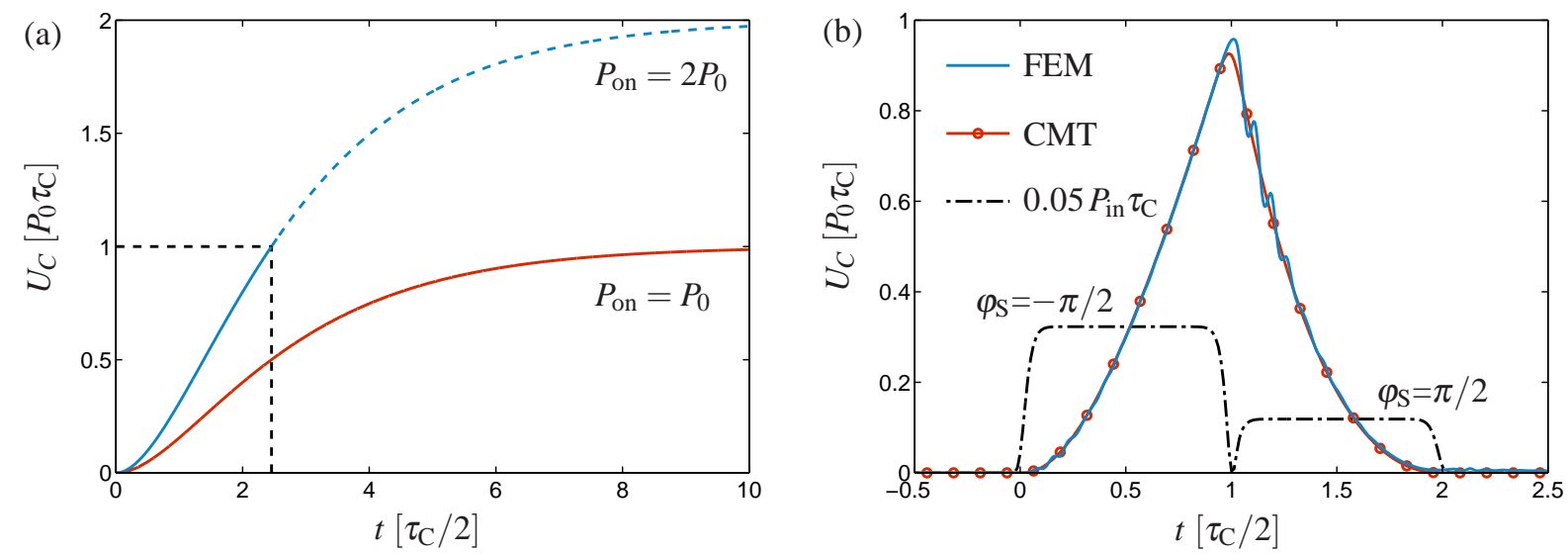

Fig. 2: (a): Cavity energy variation $U_{\mathrm{C}}(t)$ for different constant input powers as indicated. Vertical dashed line indicates the improvement in switching time for reaching $U_{\mathrm{on}}=P_{0} \tau_{\mathrm{C}}$ (horizontal dashed line) when doubling the input power from $P_{\mathrm{on}}=P_{0}$ to $P_{\mathrm{on}}=2 P_{0}$. (b): Example of the two-pulsed on/off scheme for fast switching based on two input pulses (black dashed) with different phases as indicated. The CMT calculations (red dashed with circles) compare well to independent FEM calculations (blue solid), confirming the applicability of the switching scheme. 


\section{Optimal switching}

Since in the CMT the switching can be made arbitrarily fast, the relevant quantity to optimize is not the switching time, but rather the integrated input power required to perform the switching action in a certain time. Thus, for a given switching time $\Delta t=t_{2}-t_{1}$ and given $U_{\mathrm{C}}\left(t_{1}\right)$ and $U_{\mathrm{C}}\left(t_{2}\right)$, we seek the function $P_{\text {in }}(t)$ that minimizes the integral

$$
U_{\text {Switch }}=\int_{t_{1}}^{t_{2}} P_{\text {in }}(t) \mathrm{d} t,
$$

while maintaining the correct phase relation at all times. In practice, the boundary conditions $U_{\mathrm{C}}\left(t_{1}\right)$ and $U_{\mathrm{C}}\left(t_{2}\right)$ are given by the required energy in the cavity to switch between the "on and "off state as discussed in the introduction. Eq. (5) is a classical optimization problem that we can solve analytically in the general non-linear case of Eqs. (1) and (2) using calculus of variations to find the optimum cavity energy variation $U_{\mathrm{C}}^{\mathrm{opt}}(t)$. Eqs. (1) and (2) subsequently provide the appropriate input power and phase as a function of time. As an example we consider again the switching problem from section 2 in which case the optimal cavity energy variation and input power variation are shown in Fig. 3. Within the validity of CMT, this represents the optimal way of switching the cavity energy via the two-pulsed on/off scheme. This should be compared to the constant power scheme in section 2 which is slightly different.

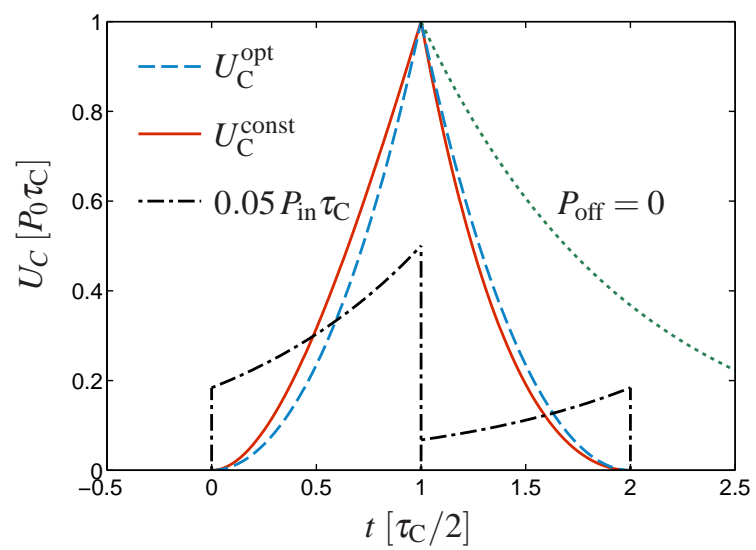

Fig. 3: Cavity energy variations in the optimal (blue dashed) and constant power (red full) schemes. Black dasheddotted lines show the (scaled) optimum input power variation. Green dotted line shows the switch off without applied power, in which case the energy leaks out at a rate of $\Gamma=2 / \tau_{\mathrm{C}}$.

\section{Conclusion}

We have presented an explicit two-pulsed on/off approach to fast switching by coherent control which, to the best of our knowledge, has not been previously suggested. The applicability of the scheme has been verified by independent numerical calculations, and based on the underlying model we have subsequently optimized the switching scheme using calculus of variations.

This work was supported by the EU FP7 project "Copernicus" and the Danish Council for Independent Research.

\section{References}

1. S. Sandhu, M. L. Povinelli and S. Fan, "Enhancing optical switching with coherent control", Appl. Phys. Lett. 23, 231108 (2010).

2. P. T. Kristensen, M. Heuck and J. Mørk, "Coherent all-optical switching in a bistable waveguide-cavitywaveguide system", CLEO/Europe and EQEC 2011 Conference Digest, paper CI P1 (2011).

3. M. Heuck, P. T. Kristensen and Jesper Mørk, "Energy-bandwidth trade-off in all-optical photonic crystal microcavity switches", Opt. Express 19, 18410 (2011).

4. J. D. Joannopoulos, S. G. Johnson, J. N. Winn and R. D. Meade, Photonic Crystals - Molding the Flow of Light, 2nd Ed. (Princeton 2008).

5. M. Soljačić and J. D. Joannopoulos, "Enhancement of nonlinear effects using photonic crystals" Nature Mater. 3, 211219 (2004) 